\title{
POLÍTICAS ASISTENCIALES PARA REZAGOS ESTRUCTURALES: EL CASO DE CHIAPAS
}

\author{
Julieta Martínez Cuero* \\ Óscar Peláez Herreros**
}

RESUMENः Con el objetivo de develar las causas del buen desempeño de los índices de marginación y rezago social durante los últimos años en Chiapas, se analizan en profundidad los componentes de estos indicadores, encontrando que, más allá de una presentación simplista de sus cifras principales, los problemas estructurales de la entidad persisten. Los progresos se han dado en aspectos con escaso impacto en el proceso de desarrollo. Apenas ha habido mejoras en los mecanismos de generación de ingresos o en la educación de los residentes. Por ello, se concluye que los avances recientes no son auto-sostenibles y que necesitan ser complementados con políticas enfocadas a la remoción de los problemas histórico-estructurales que aún padece la entidad. No obstante, se advierte de la falta de incentivos para efectuar este cambio en la orientación de las políticas públicas.

PALABRAS CLAVEः pobreza, marginación, dependencia, cambio estructural, causación acumulativa.

* Docente de la Universidad Tecnológica de México y consultora independiente, México.

** Profesor-investigador de El Colegio de la Frontera Norte, sede Tijuana, México. 
ABSTRACT: With the intent to uncover the causes of improved performance in the indices of marginalization and social exclusion in recent years in Chiapas, this article performs a deep analysis of the components that make up these indicators, finding that -beyond a simplistic presentation of the key figures- structural problems in the state persist. Gains have been made in some respects with little impact in the overall development process. There have barely been any improvements in the mechanisms to generate new incomes or in the education of residents. Therefore, it concludes that recent advances are not self-sustaining and there is a need to complement these efforts with focused policies to address historical-structural problems that remain. However, it warns of a lack of incentives to bring about that change in the application of public policies.

KEY WORDS: poverty, marginalization, dependency, structural change, cumulative effects. 


\section{INTRODUCCIÓN}

finales de 2011, la publicación de las cifras de pobreza multidimen-
sional (Coneval, 2011a), rezago social (Coneval, 2011b) y margina-
ción (Vega et al., 2011) evidenció un avance sustancial en las condiciones de vida de la población chiapaneca, al menos en los aspectos sintetizados por estos indicadores. El hecho de que Chiapas se situara entre las entidades con mayor reducción en el número de pobres o que dejara de ser el estado con mayor rezago social constituyó un éxito de suma importancia para una entidad que en 2000 estaba clasificada como la peor del país en términos de los índices de marginación (Ávila et al., 2001), rezago social (Coneval, 2011b) y desarrollo humano (PNUD, 2003; PNUD, 2012), que contaba con la mitad de sus habitantes en situación de pobreza alimentaria (Coneval, 2008: 23) y que había llegado a incluir en la Constitución local el cumplimiento de los Objetivos de Desarrollo del Milenio (oDM) promovidos por la Organización de Naciones Unidas (ONU).

En esta investigación se presta atención a la efectividad de la política social en el combate a la marginación, el rezago social y la pobreza, así como a la mejora de las condiciones de vida de la población de Chiapas con el objetivo de develar las causas de los avances mencionados y estudiar su sostenibilidad a medio y largo plazo. La hipótesis de trabajo es que los progresos advertidos se deben a cambios en variables que afectan directamente a los indicadores, pero que no modifican la estructura productiva ni mejoran los mecanismos de generación de riqueza de la población residente, de manera que no son auto-sostenibles, sino que para su consolidación y futuro avance dependen de la continua expansión del gasto público. La pobreza, la marginación y el rezago de Chiapas se explican por causas histórico-estructurales no resueltas. Las mejoras recientes responden a intervenciones específicas, muchas veces financiadas con fondos ajenos a la entidad, que lejos de actuar sobre las causas de los problemas, facilitando su remoción, incluso los acentúan al crear depen- 
dencia entre la población y la necesidad de financiación externa en el gobierno. ${ }^{1}$

Para verificar estas cuestiones, en primer lugar, se describe de manera sucinta la evolución de la política social mexicana en perspectiva histórica, dando cuenta del abandono del componente productivo y de la adopción del asistencialismo. Seguidamente, se presenta la situación de Chiapas en ese contexto, poniendo especial énfasis en el hecho de que, aún en 2010, el 42.76 por ciento de la población ocupada en la entidad trabajaba en el sector primario (INEGI, 2011). A continuación, se describen los conceptos y planteamientos teóricos que sostienen el argumento de la investigación. Posteriormente, se analizan los indicadores oficiales, encontrando ritmos de progreso diferentes en las dimensiones que los componen. Las conclusiones consecuentes se exponen en el último apartado.

\section{EVOLUCIÓN DE LA POLÍTICA SOCIAL EN MÉXICO: \\ DEL IMPULSO A LA PRODUCCIÓN AL ASISTENCIALISMO}

La política social en México ha tenido distintas etapas asociadas a modificaciones en el diseño de los programas y en la identificación de la población objetivo en función de la coyuntura política y económica del país, pero también del entorno internacional. A lo largo del último medio siglo, el modelo de desarrollo nacional ha cambiado de manera sustancial junto con las prioridades a las que atiende.

Al inicio de la fase de industrialización por sustitución de importaciones (ISI), entre 1940 y 1970, la política económica descansaba en la expansión de la agricultura y en la promoción industrial. En estos años, se impulsó la producción agropecuaria y el desarrollo de las áreas rura-

${ }^{1}$ El propio gobernador justificó públicamente la deuda financiera acumulada en el sexenio 20062012 por la necesidad de saldar «la enorme deuda social heredada», dando por bueno el desequilibrio presupuestario a cambio de no ocupar el último lugar en el indicador de rezago social ( $E l$ Heraldo de Chiapas, 2012b; López y Mayo, 2012: 56). 
les mediante programas de subsidios y compras inducidas por el Estado. Con estas medidas se buscaba el fortalecimiento del mercado interno y la creación de una base económica sólida para la industrialización del país. Por este motivo, los recursos públicos se canalizaron primordialmente hacia los productores agrícolas a gran escala, excluyendo a aquellos con producciones de subsistencia.

Cuando el modelo isi empezó a agotarse, el campo y sus habitantes sintieron las consecuencias de manera especial. La densidad poblacional de las áreas rurales aumentó a causa de la transición demográfica, expandiéndose el número de unidades de producción campesinas a pesar de las serias limitaciones de producción y comercialización que empezaban a enfrentar (Carton de Grammont, 2009). Las condiciones de vida de los agricultores se deterioraron gravemente al tiempo que las ciudades eran incapaces de absorber los excedentes de mano de obra del sector primario. Como menciona Ordóñez (2002), desde ese momento, la pobreza se volvió un problema intrínseco a las zonas rurales.

A partir del agotamiento de la ISI, Barajas (2002) distingue tres etapas en lo que denomina "políticas de administración de la pobreza en México». La primera etapa, de 1970 a 1982, se caracteriza por el diseño y puesta en funcionamiento de programas enfocados a sostener el consumo y la producción en áreas de bajo potencial económico con el objeto de conservar el control político de la población y mantener la paz social. En estos años se destinó gran cantidad de recursos al campo y a los sectores de mayor vulnerabilidad, pero sin una estrategia sostenible a largo plazo. El Programa de Inversión para el Desarrollo Rural (Pider) y El Plan Nacional de Zonas Deprimidas y Grupos Marginados (Coplamar) se encargaron de canalizar las ayudas.

En la medida en que el alza de los precios del petróleo desvió la atención hacia la promoción y desarrollo de esta actividad extractiva, la política social fue orientándose al asistencialismo, haciéndose característico el derroche de recursos públicos (Bazdresch, 1989). El cambio de política económica, en general, resultó crítico para los sectores agropecuario e industrial y para el equilibrio y diversificación de la estruc- 
tura productiva del país, que acabó atrapado en la crisis de la deuda (Griffith-Jones y Sunkel, 1986), la década perdida, la «inevitabilidad» del modelo neoliberal (Brieger, 2002).

A partir de la crisis económica de 1982, se inició una fase de abrupta apertura comercial y desregulación financiera, en la que el Estado abandonó las políticas de bienestar social para todos los mexicanos, comprometiéndose sólo a combatir la pobreza extrema en las zonas de más alta marginación y vulnerabilidad. Tetreault (2012) caracteriza a esta etapa como un periodo de reorientación de la política social mexicana, en la que el mercado pasa a ser visto como el único mecanismo capaz de generar crecimiento económico y desarrollo. De 1971-1982 a 1983-1988, el gasto social redujo su participación en el рів de 7.6 a 6.7 por ciento (Zepeda, 1994: 172).

El sexenio 1988-1994 sirvió para reforzar los cambios emprendidos en el anterior. La política social se ajustó a la «racionalidad de los recursos públicos» dictada desde los organismos internacionales a través de la disciplina fiscal. Las políticas sociales, incluyendo las enfocadas al empleo, quedaron subordinadas al ajuste estructural y a la estabilización económica del país (Ceja, 2004). La pobreza fue concebida como un fenómeno predominantemente rural y expresado en problemas de desnutrición, insalubridad, mortalidad infantil y analfabetismo. En estos años, bajo el esquema de fortalecimiento de los instrumentos de coordinación entre los diferentes niveles de gobierno, surgió el Plan Nacional de Desarrollo y el Estado abandonó el papel de benefactor. El Programa Nacional de Solidaridad (Pronasol) se encargó de focalizar su acción en zonas de alta marginación, orientándose a indígenas, campesinos y ciertos grupos urbanos excluidos de otros programas. El trato a la población objetivo cambió notablemente. A pesar del conocimiento del problema de la pobreza rural, se abandonó la política de generación de empleo en el campo, se eliminaron los precios de garantía de los granos básicos, desaparecieron los programas de compras gubernamentales y se redujeron los créditos destinados a la producción y a la comerciali- 
zación (Calva, 1993). Todo ello resultó en una severa caída de los salarios reales en el campo mexicano.

La etapa emprendida en 1995 no implicó grandes variaciones respecto a la anterior. La política social siguió subordinada a los ajustes de la economía nacional determinados en el contexto internacional. Se continuó creyendo que la pobreza era un problema soluble a través de la eficiencia del mercado y que el incremento de la inversión privada promovería el bienestar social (Levy, 1991). Los cambios más importantes se dieron en la administración y en los mecanismos de asignación de los recursos públicos encaminados a combatir la pobreza. Se buscó la construcción de una política integral de desarrollo social orientada por los principios del federalismo, iniciándose la descentralización hacia estados y municipios mediante la captación de recursos federales en el ramo 33. Los programas de Solidaridad en la Alianza Nacional para el Bienestar, Programa de Educación, Salud y Alimentación (Progresa) y el programa para superar la pobreza tomaron protagonismo en este periodo, que concluyó con cifras de pobreza superiores a las observadas antes de la crisis de 1995 (Acosta, 2010: 241).

El cambio de signo político que tuvo lugar en 2000 se asoció a los principios de justicia y democracia social, abriendo la posibilidad de desarrollar líneas de acción para atender las necesidades de la población más vulnerable y excluida por los anteriores gobiernos y programas. A pesar de ello, y de la aprobación en 2004 de la Ley General de Desarrollo Social, en la práctica las acciones se alinearon al marco precedente, sin rebasar la línea del asistencialismo. El Programa de Desarrollo Humano Oportunidades se conformó, en lo esencial, a semejanza de Progresa, complementándose con los programas de asistencia alimentaria y nutricional ya vigentes.

A partir de la elección de 2006, se insistió en atender las carencias de educación, salud, vivienda y alimentación mediante el programa Vivir Mejor. En el terreno de la producción, ante una situación de escasez e incremento de los precios internacionales de los granos básicos, se puso en marcha el Programa de Acciones de Apoyo a la Economía Familiar 
que, en la práctica y en los resultados, no alcanzó a impulsar la productividad del campo mexicano.

\section{CAUSAS HISTÓRICO-ESTRUCTURALES \\ DE LA POBREZA EN CHIAPAS}

La estructura productiva de Chiapas se basa en el sector primario. En 2010, 95 de los 118 municipios entonces existentes concentraban más ocupados en este sector que en cualquiera de los dos restantes. En términos agregados, 42.76 por ciento de los trabajadores de Chiapas se desempeñaban en actividades primarias (cuadro 1 ); una cifra muy alejada de la media nacional (13.36 por ciento), pero también de la propia de la segunda entidad con mayor proporción de trabajadores primarios, Oaxaca (32.41 por ciento).

\section{CUADRO 1}

Porcentaje de ocupados por sector de actividad (\%)

\begin{tabular}{lllll}
\hline CHIAPAS & PRIMARIO & SECUNDARIO & TERCIARIO & NO ESPECIFICADO \\
\hline 1990 & 58.34 & 11.13 & 27.43 & 3.11 \\
2000 & 47.25 & 13.24 & 37.31 & 2.20 \\
2010 & 42.76 & 13.51 & 42.90 & 0.83
\end{tabular}

\begin{tabular}{lllll}
\hline MÉXICO & PRIMARIO & SECUNDARIO & TERCIARIO & NO ESPECIFICADO \\
\hline 1990 & 22.65 & 27.79 & 46.13 & 3.43 \\
2000 & 15.83 & 27.82 & 53.35 & 3.00 \\
2010 & 13.36 & 24.44 & 60.88 & 1.32 \\
\hline
\end{tabular}

Fuente: Elaboración propia con datos de INEGI (1993, 2003 y 2011).

Con el paso del tiempo, la participación laboral en el sector primario se ha reducido en respuesta a la industrialización y terciarización de la economía (Chenery y Syrquin, 1978: 68-71). A pesar de que este pro- 
ceso también se observa en el caso de Chiapas, en 2010, el porcentaje de ocupados primarios aún duplicaba el valor medio nacional de 1990. Una diferencia fundamental es que en Chiapas se está dando una terciarización sin industrialización previa. La participación de los ocupados en el sector secundario tiende a mantenerse estable a lo largo del tiempo, siendo algo inferior a la mitad de la media nacional. La leve alza que se advierte en los datos de la entidad, especialmente entre 1990 y 2000 (cuadro 1), responde al aumento del empleo en el subsector de la construcción.

En cualquier caso, el problema de la estructura ocupacional no es tanto el predominio de las actividades primarias, como el tipo de agricultura, industria y servicios que dan empleo a la mayoría de los chiapanecos. En 2010, 69.85 por ciento de la población ocupada recibía menos de dos salarios mínimos. Oaxaca, la siguiente entidad en esta clasificación, apenas llegaba a 57.77 por ciento. En todo el país, la cifra era de 38.66 por ciento (Vega et al., 2011:26).

La situación de pobreza extrema que afecta a un tercio de los chiapanecos (Coneval, 2013) es un problema con raíces histórico-estructurales, relacionado con el desarrollo de las fuerzas productivas y el acceso a los medios de producción. La población sigue laborando en el sector primario porque no dispone de mejores opciones, a pesar de que, en la actualidad, esta actividad sólo garantiza la subsistencia al complementarse con transferencias del gobierno. El origen del rezago social, la marginación y la pobreza ha de buscarse, por tanto, en el reparto de las fuerzas productivas y los frutos del trabajo, para lo cual el control y acceso a la tierra se constituyen en factores claves al ser parte esencial de la secuencia que ha llevado a la actual situación.

En el caso específico de Chiapas, el grueso de la población ha permanecido al margen de los beneficios logrados en las distintas fases de lucha nacional por el reparto de la tierra. Según Tarrío y Concheiro (2006), la Independencia de México no representó cambios significativos en las condiciones de vida de los chiapanecos. Hacia 1820, los poseedores de tierras seguían siendo la Iglesia, los hacendados y los fin- 
queros, que ejercían el poder político y económico del Estado. En la etapa porfirista la situación empeoró con el despojo de tierras a las comunidades indígenas. Grandes extensiones territoriales fueron vendidas a extranjeros que se beneficiaron de la fertilidad del suelo y de la disponibilidad de fuerza de trabajo barata. Durante la reforma agraria, el reparto de tierra se realizó de manera lenta y marginal, conjugando intereses económicos y políticos. De acuerdo con Núñez (2004), la política de dotación de tierras dependió de las coyunturas políticas y de los intereses particulares de cada una de las regiones de Chiapas. En la región Altos, por ejemplo, se excluyó del reparto a miles de indígenas que se encontraban trabajando en las fincas cafetaleras del Soconusco o en las plantaciones de maíz de las regiones bajas del estado. El fallido reparto agrario impidió que los campesinos chiapanecos se beneficiaran de las políticas gubernamentales enfocadas a estimular la producción, ya que estos programas se concentraron en los productores a gran escala.

En fechas más recientes, en concreto entre 1991 y 2007, el número de unidades de producción dedicadas a actividades agrícolas y ganaderas aumentó 32.7 por ciento, periodo en el que la superficie agrícola incluso se redujo 23 por ciento (INEGI, 1994 y 2009). El crecimiento de la población sobre una base material inamovible ha resultado en la atomización de los recursos productivos. Súmese a ello la falta de tecnología y modernización de las técnicas de cultivo. El 97.9 por ciento de la superficie con actividad agropecuaria sigue sembrándose en condiciones de temporal, y sólo 1.9 por ciento de las unidades de producción ha recibido capacitación en cuestiones de agricultura durante los últimos diez años. De este modo, un problema histórico no resuelto, como es la estructura semi-feudal de la propiedad de la tierra, unido al incremento de la población, ha resultado en deterioro ambiental, pérdida de fertilidad de la superficie de labor, mínimos niveles de productividad y altos porcentajes de pobreza.

Si el sector primario acumula estas deficiencias y dificultades crecientes, ¿por qué la población no busca actividades alternativas? Dos 
aspectos clave ayudan a responder esta pregunta: los bajos niveles de educación, que limitan la movilidad profesional, y el hecho de que las perspectivas en el resto de sectores no sean mucho más prometedoras. No obstante, hay que reconocer que la población está abandonando lentamente el sector primario (cuadro 1), pero también la entidad en busca de las oportunidades que se le niegan en su tierra (López y Peláez, 2013).

Respecto a los niveles de educación, basta señalar que, desde 1980, Chiapas es la entidad del país con mayor porcentaje de población analfabeta de 15 años y más. El caso es que en 1970 no lo era: Guerrero y Oaxaca la superaban (Aparicio, 2004: 52). Al inicio de la década pasada, Sánchez (2000ः 104) ya ponía de ejemplo los «severos deterioros» que se habían registrado en los indicadores educativos de Chiapas. A pesar de la advertencia, en el periodo 2000-2010, la tasa de analfabetismo de la entidad no sólo ha marcado el máximo nacional, sino que, además, ha experimentado el menor ritmo de mejora de toda la federación.

El producto por trabajador del sector primario es el segundo más bajo de todo el país, superando únicamente al de Guerrero. ${ }^{2}$ Lo mismo ocurre en el sector terciario, donde la productividad del trabajo de Chiapas sólo se sitúa por encima de la de Oaxaca. La única rama de actividad con una producción por trabajador superior a la media nacional es la «industria extractiva y de la electricidad», basada en explotaciones de enclave con mínimos eslabonamientos en la entidad. ${ }^{3}$ En conjunto, el pro-

${ }^{2}$ El producto por trabajador, o productividad del trabajo, se calcula a partir de las cifras del pIB por actividad económica y entidad federativa (a precios constantes de 2008) obtenidas del Sistema de Cuentas Nacionales de México, y de los datos de población ocupada por actividad de la Encuesta Nacional de Ocupación y Empleo publicados por INEGi. Los resultados que se comentan corresponden a 2012, el más reciente para el cual se dispone de información en ambas variables.

${ }^{3}$ Como explican López et al. (2009: 42), esta rama apenas tiene impacto sobre la economía chiapaneca ya que los recursos correspondientes son explotados por empresas paraestatales y captados por las finanzas centrales. La mano de obra especializada (técnicos, ingenieros) no es local, sino importada de otros estados, lo que dificulta que sus altos salarios se gasten en Chiapas, anulando, prácticamente, su efecto multiplicador sobre la economía de la entidad. 
ducto por trabajador de la economía chiapaneca $(\$ 118,298)$ es el más bajo de todo el país $(\$ 262,030)$, lo que da idea de la reducida capacidad para generar ingresos.

A pesar de los avances, en 2010, Chiapas era la entidad federativa con mayor porcentaje de población ocupada que no alcanzaba a ganar dos salarios mínimos (Vega et al., 2011:57). Los datos de la Encuesta Nacional de Ocupación y Empleo (ENOE) confirman que en el último trimestre de 2013 la posición de Chiapas seguía invariada en este aspecto. Además, era la entidad con mayor tasa de condiciones críticas de ocupación (34.3 por ciento), a gran distancia de la media nacional (11.9 por ciento), pero también de la siguiente entidad: Tlaxcala (18.1 por ciento); clasificando en segundo lugar en las tasas de informalidad laboral y de trabajo asalariado, tras Oaxaca en ambos casos. Esta debilidad del mercado laboral dificulta seriamente las posibilidades de generar ingresos por parte de aquellos que únicamente disponen de su trabajo como fuente de riqueza.

\section{EL DESARROLLO DEL SUBDESARROLLO Y EL COMBATE A LA MARGINACIÓN Y AL REZAGO SOCIAL}

El desarrollo, como "proceso dual de enriquecimiento y cambio estructural» (Barkin, 1972: 74), implica modificaciones en los patrones de consumo-inversión, producción y comercio (industrialización y terciarización), residencia (urbanización), educación, mortalidad, etcétera (Chenery y Syrquin, 1978). Frank (1967: 165) argumenta que «el subdesarrollo ha sido y es aún generado por el mismo proceso histórico que genera también el desarrollo económico: el desarrollo del propio capitalismo», y que «nuestra ignorancia de la historia de los países subdesarrollados nos lleva a aceptar que su pasado y hasta su presente se asemejan a las etapas primitivas de la historia de los países hoy desarrollados». Una diferencia esencial es que las poblaciones menos desarrolladas (o subdesarrolladas) han accedido a los 
medicamentos, vacunas e insecticidas que limitan la incidencia de muchas enfermedades a un costo relativamente bajo, lo que ha reducido la mortalidad (Davis, 1956; Arriaga y Davis, 1969), dando lugar a transiciones demográficas caracterizadas por crecimientos explosivos de la población (Chesnais, 1992). El problema del asunto es que estas transiciones, iniciadas por motivos exógenos, han estado desligadas del resto de cambios estructurales. Con mayor crecimiento demográfico y menor progreso en las estructuras productivas, las áreas urbanas han sido incapaces de absorber los excedentes de población rural, dando lugar a enormes bolsas de pobreza.

Myrdal ([1957] 1979: 24) sostiene que el proceso de desarrollosubdesarrollo responde a fenómenos de causación circular: «un cambio no da lugar a cambios compensadores, sino que, por lo contrario, da lugar a cambios coadyuvantes que mueven al sistema en la misma dirección que el cambio original, impulsándolo más lejos. Esta causación circular hace que un proceso social tienda a convertirse en acumulativo». Advierte que el proceso acumulativo puede darse en dos direcciones: $a$ ) en sentido «positivo», a modo de «espiral ascendente» que lleva a un desarrollo que Frank (1967: 164) calificaría como «auto-perpetuado»; y b) en sentido «negativo», como «círculo vicioso» que conduce a un subdesarrollo también «auto-perpetuado», ya que, «en el proceso acumulativo, la pobreza se transforma en su propia causa» (p.47).

No obstante, el planteamiento de Myrdal ([1957] 1979) admite la posibilidad de realizar intervenciones para revertir la dirección de un proceso acumulativo negativo y reconducirlo hacia el desarrollo:

La hipótesis de la causación circular, que constituye la doctrina de la desesperación de los países más pobres mientras dejen que las cosas sigan su curso natural, puede convertirse en recompensa valiosa cuando se ponga en práctica una política de intervenciones intencionales. Si éstas se aplican a un esfuerzo nacional con metas definidas los resultados compensarán con creces los sacrificios que entrañen, siempre que ese esfuerzo contribuya a iniciar un proceso acumulativo ascendente (Myrdal ([1957] 1979): 100). 
Una medida aplicada en un punto del sistema puede iniciar el movimiento ascendente al desencadenar un proceso acumulativo de interacción en el que el cambio experimentado por un factor estaría apoyado de manera continua por la reacción de otro factor, y así sucesivamente en forma circular. El propio Myrdal ([1957] 1979: 30) advierte que no todas las medidas tienen el mismo impacto: «un aumento del empleo elevará casi inmediatamente ciertos niveles de vida; pero un cambio en los niveles de educación o de salud se alcanzará en forma más lenta, y sus efectos sobre los otros factores se demorarán, con lo que se retardará el proceso acumulativo.»

A efectos del presente análisis, cabe distinguir tres tipos de intervenciones: a) las que afectan de manera rápida (o inmediata) a la estructura productiva y a la capacidad de generar ingresos por parte de la población; b) las que favorecen de manera lenta el progreso de los residentes, y c) aquellas otras que no resuelven los problemas estructurales sino que incluso pueden contribuir a agravarlos al crear dependencia. Entre las primeras medidas se encuentran las que dinamizan el mercado de trabajo, generando más empleos mejor retribuidos, lo que incrementa el ingreso de la población que, por sí misma, puede adquirir los bienes y servicios o realizar las inversiones más convenientes para mejorar su bienestar. Entre las segundas, como hace Myrdal, pueden incluirse los progresos en salud y educación, que requieren de más tiempo para realizarse, pero que contribuyen a mejorar las condiciones de vida de la población, tanto directa como indirectamente, al facilitar también el acceso o la creación de mejores puestos de trabajo, la obtención de mayores ingresos, etcétera (Mincer, 1958; Schultz, 1961; Becker, 1964). Llegado este punto, es conveniente destacar que el ingreso, la salud y la educación son las únicas tres dimensiones que incorpora el índice de desarrollo humano (IDH) que el Programa de las Naciones Unidas para el Desarrollo (PNUD) viene publicando desde 1990 en su intento por operacionalizar el enfoque de capacidades de Sen (1985). 
En México, el IDH se ha calculado para las entidades federativas y municipios del país (PNUD, 2008 y 2012), siendo la base para la elección de los beneficiaros de la Estrategia Integral para la Atención a los $\mathrm{Mu}$ nicipios con Menor Índice de Desarrollo Humano implementada por el Gobierno Federal desde enero de 2007. No obstante, el Consejo Nacional de Población (Conapo) también calcula el índice de marginación (гм) y el Consejo Nacional de Evaluación de la Política de Desarrollo Social (Coneval) los porcentajes de pobreza y el índice de rezago social (IRs) a nivel de entidades federativas, municipios, localidades y áreas urbanas, siendo estos indicadores los utilizados para seleccionar las Zonas de Atención Prioritaria (Dof, 2013).

Aunque la marginación, el rezago social, la pobreza y el desarrollo humano son conceptos distintos, que se hacen operativos mediante técnicas y variables diferentes, compiten entre sí por convertirse en los instrumentos oficiales para el diseño, asignación y evaluación de las políticas públicas. De hecho, a pesar de sus diferencias, los resultados que arrojan tienden a asemejarse. Allí donde la marginación es mayor, también lo son el rezago social y la pobreza, mientras que el desarrollo es menor (Coneval, 2007: 495-505; Peláez, 2012).

El problema que surge con estos indicadores es que invitan a una falsa asimilación entre avances en los índices y mejoras en los conceptos representados. Se olvida que los indicadores no son más que operacionalizaciones simplificadas y abstractas, lo más precisas posibles, pero incompletas, de los conceptos, y que no toda la realidad está representada en los indicadores. El problema se agrava cuando se invierte el sentido de la relación causal que conecta (de manera imperfecta) la realidad con su reducción matemática. A pesar de que lo relevante son las mejoras en términos de bienestar (marginación, rezago o desarrollo), y no la búsqueda directa de dinámicas favorables del IM, IRS O IDH, existe la tendencia a actuar preferentemente sobre la parte de la marginación, el rezago y el desarrollo contenida en los índices, y en especial sobre aquellas variables que son más fáciles de modificar y que suelen coincidir con las que menos impacto tienen en el proceso de acumulación circular. 
El im, por ejemplo, está conformado por nueve variables observables entre las que se encuentran los porcentajes de ocupantes en viviendas sin drenaje ni excusado, ocupantes en viviendas con piso de tierra, población de 15 años o más analfabeta y población ocupada con ingreso de hasta dos salarios mínimos. No cabe duda de que una mejora en cualquiera de estas variables eleva el bienestar de la población y favorece una evolución positiva del iм a corto plazo. Sin embargo, no todas tienen la misma fuerza para impulsar y retroalimentar el proceso de desarrollo. Una familia con ingresos superiores a dos salarios mínimos dispone de más posibilidades de auto-costear una vivienda con drenaje, agua entubada, electricidad, piso firme, etcétera, que otra familia con menores ingresos y varios integrantes analfabetos, que difícilmente va a disfrutar de estos servicios y, aunque el gobierno se los proporcione, no va a poder mantenerlos por sí misma. Estas intervenciones son las que se han clasificado como $c$ ), es decir, aquellas que no resuelven los problemas estructurales sino que incluso pueden contribuir a agravarlos al crear dependencia.

Malthus explicaba que la única forma en que un pobre puede mantenerse y conservar su independencia es trabajando, ganando la subsistencia por sus medios y no dependiendo de la caridad de los demás:

[...] el hombre que efectúa una jornada de trabajo para mí no tiene por qué sentirse más obligado hacia mí que yo hacia él. Yo poseo lo que él necesita, él lo que yo necesito. Hacemos un intercambio amistoso. El pobre se mantiene erguido, consciente de su independencia; y el espíritu del patrono no se encuentra viciado por una sensación de poder. [...] [N]o disfrutaríamos hoy de nuestro actual grado de libertad civil si los pobres no hubiesen tenido, merced a la introducción de las manufacturas, la posibilidad de ofrecer algo a cambio de lo que recibían de los grandes señores en vez de tener que depender de su generosidad (Malthus, [1798] 1984: 217).

Mauss ([1924] 2002:113-114) también percibe la dependencia así como la degradación implícita en los casos de relaciones asimétricas: 
«El dar es signo de superioridad, de ser más, de estar más alto, de magister; aceptar sin devolver o sin devolver más, es subordinarse, transformarse en cliente y servidor, hacerse pequeño, elegir lo más abajo (minister)»; ${ }^{4}$ especificando que: «La caridad, todavía hoy, es hiriente para quien la acepta, tendiendo todos los esfuerzos de nuestra moral por suprimir el patronazgo inconsciente e injurioso del rico "limosnero"» (Mauss, [1924] 2002: 103). Valenzuela (2013: 40) señala:

[... el gasto estatal de ayuda a los pobres [...] opera como una limosna pública pues no crea actividades y trabajos productivos. Las funciones más o menos latentes de estos gastos apuntan a la mantención del statu-quo, evitando las posibles explosiones sociales. De hecho, [en el caso específico de Chiapas,] es muy claro que el crecimiento de este gasto social respondió al peligro del zapatismo y para nada a un real propósito de desarrollo económico regional. ${ }^{5}$

A partir de lo mencionado, cabe aclarar que no se está argumentando que el Gobierno deba abstenerse de facilitar estos servicios a las poblaciones que carecen de ellos. Lo que se plantea es que debe proveerlos como complementos, no como sustitutos, de políticas que corrijan las deficiencias y obstáculos detectados en las estructuras productiva y social, y que lastran el desarrollo de ciertas comunidades. Como se ha explicado, ésta es la forma en que pueden tener mayor impacto a medio y largo plazo. En palabras de Acosta (2010: 244):

Aunque la focalización es una estrategia racional cuando los recursos públicos son escasos, los enfoques focalizados y asistencialistas alcanzan rápidamente sus límites si las capacidades de los sujetos no son convertidas en oportunidades de desarrollo individual y colectivo y si las estructuras sociales y normativas en las que se sustenta la desigualdad social no son modificadas.

\footnotetext{
${ }^{4}$ Bartra (2007: 340) sugiere la forma en que los carentes de recursos devuelven la ayuda pública al referirse a Chiapas como "un lugar donde el voto sigue siendo para muchos un "valor de cambio" ».

${ }^{5}$ Burguete y Leyva (2004 y 2007) defienden una idea semejante.
} 


\section{DISCUSIÓN DE LOS RESULTADOS OFICIALES}

La comparación de los valores del im y el IRs a lo largo del tiempo es válida en términos relativos, pero no absolutos, debido a la técnica que se utiliza para su cómputo (Gutiérrez y Gama, 2010). Por ejemplo, para el caso de Chiapas, lo relevante no es tanto que mejoren las variables que integran estos indicadores, como que mejoren más que en otras entidades federativas. Sólo así es posible registrar reducciones del i m o del IRs. La interpretación «Chiapas deja de ser el estado con mayor rezago social» es técnicamente correcta. Falta ver los detalles que han llevado a esta evolución del índice.

En el cuadro 2 se muestra que el Irs de Chiapas experimentó una sensible reducción entre 2005 y 2010, desde un valor de 2.548 a 2.271. Este cambio permitió superar a Guerrero y Oaxaca. Como se observa, las once variables evolucionaron de manera favorable entre estas fechas, algo que también ocurrió en el periodo 2000-2005, sólo que entonces las mejoras no fueron suficientes para dar lugar a una reducción del rezago relativo, y el IRs incluso aumentó.

El mayor avance se observa en la variable que mide la población sin derechohabiencia a servicios de salud, en la que Chiapas pasó de presentar el valor más alto de toda la federación a ocupar el quinto lugar. Cabe destacar que esta variable no se refiere a salud efectiva de la población, sino simplemente a derechohabiencia a servicios de salud. El porcentaje de viviendas que no disponen de excusado o sanitario también muestra un progreso destacado durante la década en cuestión, en la que Chiapas pasa de ser de la cuarta a la novena entidad con mayor carencia en este servicio. Las demás variables relacionadas con la calidad y servicios básicos en las viviendas presentan valores bastante satisfactorios en comparación con el resto de entidades. Los problemas se concentran en los tres indicadores de educación y en los dos de activos en el hogar, en los que Chiapas sistemáticamente clasifica como la entidad con mayor rezago del país. 
CUADRO 2

Valores del IRS y de sus componentes, y clasificación para Chiapas, 2000, 2005 y 2010

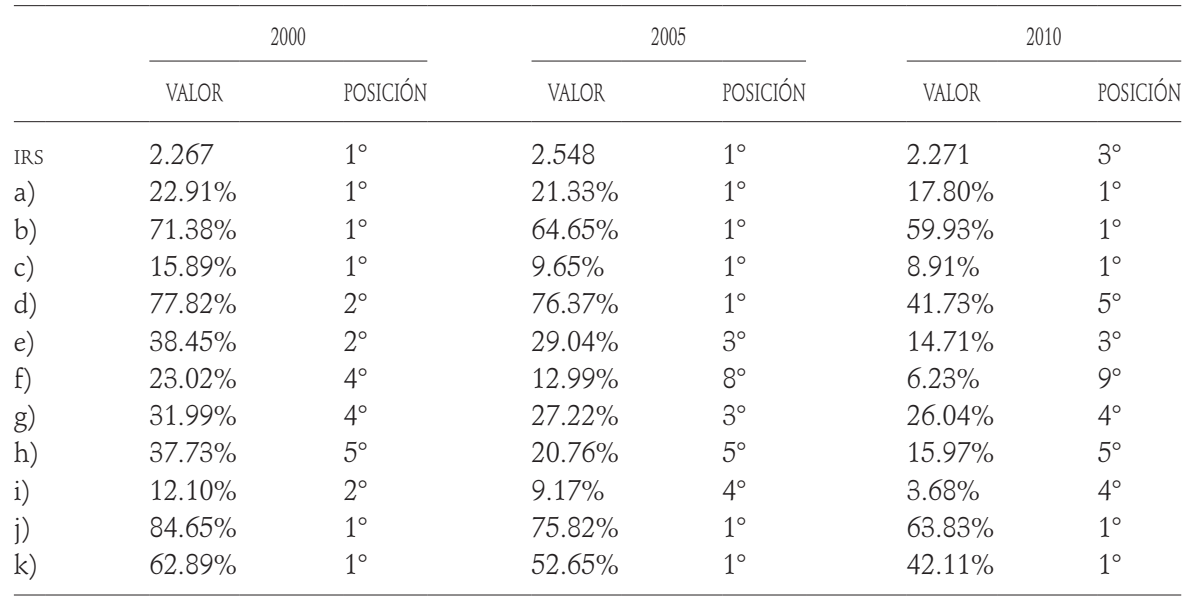

Nota: Las variables componentes del IRS son los porcentajes de: a) población de 15 años o más analfabeta; b) población de 15 años y más con educación básica incompleta; c) población de 6 a 14 años que no asiste a la escuela; d) población sin derechohabiencia a servicios de salud; e) viviendas con piso de tierra; f) viviendas que no disponen de excusado o sanitario; $g$ ) viviendas que no disponen de agua entubada de la red pública; $h$ ) viviendas que no disponen de drenaje; i) viviendas que no disponen de energía eléctrica; $j$ ) viviendas que no disponen de lavadora; $k$ ) viviendas que no disponen de refrigerador.

Fuente: Elaboración propia con datos de Coneval (2011b).

El Im permite corroborar estas tendencias y prolongarlas en el tiempo, ya que, a partir de la información recopilada por Aparicio (2004: 52-60), es posible reconstruir el índice hasta 1970 recurriendo a la técnica de componentes principales. Como se muestra en el cuadro 3, Chiapas es la entidad con Im más alto sólo en 1990, 1995 y 2000. Antes de 1990 lo era Oaxaca y después de 2000 lo es Guerrero. Las variables que mejor evolucionaron en la última década fueron los porcentajes de ocupantes en viviendas sin drenaje ni excusado (del cuarto al décimo puesto) y ocupantes en viviendas sin energía eléctrica (del segundo al quinto puesto). Las demás variables tendieron a mantener sus posiciones relativas. Los mayores problemas se concentran en cuatro variables en las 
que Chiapas clasifica persistentemente en la última posición: los dos indicadores de educación (población de 15 años o más analfabeta y población de 15 años o más sin primaria completa), viviendas con algún nivel de hacinamiento y el indicador de ingreso (población ocupada con ingreso de hasta dos salarios mínimos).

\section{CUADRO 3}

Valores del Im y de sus componentes, y clasificación para Chiapas, 1970 a 2010

\begin{tabular}{lccccccccccccccc}
\hline & \multicolumn{3}{c}{1970} & \multicolumn{2}{c}{1980} & \multicolumn{2}{c}{1990} & & 1995 & & 2000 & & 2005 & & 2010 \\
\hline IM & 1.47 & $2^{\circ}$ & 2.01 & $2^{\circ}$ & 2.36 & $1^{\circ}$ & 2.42 & $1^{\circ}$ & 2.25 & $1^{\circ}$ & 2.33 & $2^{\circ}$ & 2.32 & $2^{\circ}$ \\
a) & $45 \%$ & $3^{\circ}$ & $38 \%$ & $1^{\circ}$ & $30 \%$ & $1^{\circ}$ & $26 \%$ & $1^{\circ}$ & $23 \%$ & $1^{\circ}$ & $21 \%$ & $1^{\circ}$ & $18 \%$ & $1^{\circ}$ \\
b) & $89 \%$ & $1^{\circ}$ & $75 \%$ & $1^{\circ}$ & $62 \%$ & $1^{\circ}$ & $55 \%$ & $1^{\circ}$ & $50 \%$ & $1^{\circ}$ & $43 \%$ & $1^{\circ}$ & $37 \%$ & $1^{\circ}$ \\
c) & $77 \%$ & $7^{\circ}$ & $73 \%$ & $3^{\circ}$ & $43 \%$ & $5^{\circ}$ & $28 \%$ & $5^{\circ}$ & $19 \%$ & $4^{\circ}$ & $8 \%$ & $9^{\circ}$ & $5 \%$ & $1^{\circ}$ \\
d) & $69 \%$ & $3^{\circ}$ & $55 \%$ & $1^{\circ}$ & $35 \%$ & $1^{\circ}$ & $23 \%$ & $1^{\circ}$ & $12 \%$ & $2^{\circ}$ & $6 \%$ & $3^{\circ}$ & $4 \%$ & $5^{\circ}$ \\
e) & $62 \%$ & $3^{\circ}$ & $56 \%$ & $2^{\circ}$ & $42 \%$ & $4^{\circ}$ & $34 \%$ & $4^{\circ}$ & $25 \%$ & $5^{\circ}$ & $26 \%$ & $3^{\circ}$ & $22 \%$ & $3^{\circ}$ \\
f) & $53 \%$ & $5^{\circ}$ & $82 \%$ & $3^{\circ}$ & $74 \%$ & $1^{\circ}$ & $70 \%$ & $1^{\circ}$ & $65 \%$ & $1^{\circ}$ & $60 \%$ & $1^{\circ}$ & $54 \%$ & $1^{\circ}$ \\
g) & $69 \%$ & $3^{\circ}$ & $62 \%$ & $2^{\circ}$ & $51 \%$ & $2^{\circ}$ & $42 \%$ & $3^{\circ}$ & $41 \%$ & $2^{\circ}$ & $33 \%$ & $3^{\circ}$ & $16 \%$ & $3^{\circ}$ \\
h) & $79 \%$ & $2^{\circ}$ & $74 \%$ & $3^{\circ}$ & $67 \%$ & $2^{\circ}$ & $63 \%$ & $2^{\circ}$ & $61 \%$ & $2^{\circ}$ & $58 \%$ & $2^{\circ}$ & $58 \%$ & $3^{\circ}$ \\
i) & $75 \%$ & $1^{\circ}$ & $83 \%$ & $6^{\circ}$ & $80 \%$ & $1^{\circ}$ & $82 \%$ & $1^{\circ}$ & $76 \%$ & $1^{\circ}$ & $78 \%$ & $1^{\circ}$ & $70 \%$ & $1^{\circ}$ \\
\hline
\end{tabular}

Nota: Las variables componentes del IM son los porcentajes de: a) población de 15 años o más analfabeta; b) población de 15 años o más sin primaria completa; c) ocupantes en viviendas sin drenaje ni excusado; d) ocupantes en viviendas sin energía eléctrica; e) ocupantes en viviendas sin agua entubada; f) viviendas con algún nivel de hacinamiento; $g$ ) ocupantes en viviendas con piso de tierra; $h$ ) población en localidades con menos de 5,000 habitantes; i) población ocupada con ingreso de hasta 2 salarios mínimos.

Fuente: Elaboración propia con datos de Ávila et al. (2001), Aparicio (2004), Anzaldo y Prado (2006) y Vega et al. (2011).

A diferencia del IRs, el IM no incorpora ninguna variable relacionada con la salud. La buena evolución de esta componente contribuye a explicar el mayor avance del IRs frente al IM. El IDH, en cambio, no sólo tiene en cuenta la derechohabiencia a servicios de salud, sino la salud efectiva de la población a través de la esperanza de vida. A partir de los datos de PNUD (2012: 10-1) se puede comprobar que, en 2010, Chiapas clasificaba mejor que Guerrero y Veracruz en el subíndice de salud. No así en educación e ingreso, donde ocupa el peor puesto. En conjunto, el IDH de Chiapas sigue siendo el más bajo de toda la federación. 
La publicación por parte de Coneval (2011a) de las cifras de pobreza multidimensional, comparando la situación de 2010 con la de 2008, llevó a la conclusión de que Chiapas era una de las entidades federativas del país donde más se había reducido la pobreza extrema. En concreto, 72 mil personas habían dejado de ser pobres extremos (El Heraldo de Chiapas, 2011 y 2012a). No se mencionaba que a entidades como Aguascalientes o Baja California Sur les era imposible alcanzar esa cifra de reducción al apenas contar con 47 y 16 mil pobres, respectivamente. En cualquier caso, la información facilitada por Coneval (2013) sobre pobreza multidimensional sirve para confirmar lo comentado a partir de los otros indicadores.

\section{CUADRO 4}

Porcentajes de población en situación de pobreza multidimensional, componentes por dimensión, y clasificación para Chiapas, 2010 y 2012

\begin{tabular}{|c|c|c|c|c|}
\hline \multirow{2}{*}{ PORCENTAJES DE POBLACIÓN EN POBREZA } & \multicolumn{2}{|c|}{2010} & \multicolumn{2}{|c|}{2012} \\
\hline & VALOR & POSICIÓN & VALOR & POSICIÓN \\
\hline Multidimensional & $78.48 \%$ & $1^{\circ}$ & $74.69 \%$ & $1^{\circ}$ \\
\hline Moderada & $40.21 \%$ & $8^{\circ}$ & $42.52 \%$ & $6^{\circ}$ \\
\hline Extrema & $38.27 \%$ & $1^{\circ}$ & $32.17 \%$ & $1^{\circ}$ \\
\hline \multicolumn{5}{|l|}{$\begin{array}{l}\text { Porcentajes de población con } \\
\text { carencia: }\end{array}$} \\
\hline educativa & $35.01 \%$ & $1^{\circ}$ & $33.48 \%$ & $1^{\circ}$ \\
\hline por acceso a los servicios de salud & $35.39 \%$ & $5^{\circ}$ & $24.94 \%$ & $6^{\circ}$ \\
\hline por acceso a la seguridad social & $82.37 \%$ & $1^{\circ}$ & $83.29 \%$ & $1^{\circ}$ \\
\hline por calidad y espacios de la vivienda & $33.25 \%$ & $3^{\circ}$ & $29.15 \%$ & $2^{\circ}$ \\
\hline por servicios básicos en la vivienda & $60.69 \%$ & $1^{\circ}$ & $56.84 \%$ & $2^{\circ}$ \\
\hline por acceso a la alimentación & $30.31 \%$ & $5^{\circ}$ & $24.73 \%$ & $15^{\circ}$ \\
\hline \multicolumn{5}{|l|}{$\begin{array}{l}\text { Porcentajes de población con } \\
\text { ingreso inferior: }\end{array}$} \\
\hline \multirow{2}{*}{ PORCENTAJES DE POBLACIÓN EN POBREZA } & \multicolumn{2}{|c|}{2010} & \multicolumn{2}{|c|}{2012} \\
\hline & VALOR & POSICIÓN & VALOR & POSICIÓN \\
\hline a la línea de bienestar & $80.85 \%$ & $1^{\circ}$ & $76.42 \%$ & $1^{\circ}$ \\
\hline a la línea de bienestar mínimo & $50.89 \%$ & $1^{\circ}$ & $46.70 \%$ & $1^{\circ}$ \\
\hline
\end{tabular}


A pesar de los avances, aún en 2012, la entidad sigue presentando el mayor porcentaje de población en situación de pobreza multidimensional (74.69 por ciento), lejos de la media nacional (45.48 por ciento) e incluso de la siguiente entidad con mayor porcentaje: Guerrero (69.70 por ciento). Las carencias más graves se encuentran en educación y en el acceso a la seguridad social (cuadro 4). Las que menos problemas presentan son acceso a servicios de salud y alimentación. Los porcentajes de población con ingreso inferior a las líneas de bienestar y bienestar mínimo son los más altos de la federación, mostrando la escasa capacidad de la población residente para generar recursos.

\section{CONCLUSIONES}

En los últimos años, Chiapas ha avanzado sustancialmente en el combate de ciertos rezagos. El acceso a servicios de salud y la calidad y servicios básicos en las viviendas son los aspectos que más han mejorado, favoreciendo que la entidad deje de ocupar la última posición en el im y el irs. No obstante, dos aspectos claves para el desarrollo y el bienestar de la población, como son la educación y el ingreso, parecen haber tenido dinámicas mucho más pobres. Chiapas sigue siendo la entidad con mayor porcentaje de población de 6 a 14 años que no asiste a la escuela, de 15 y más años con educación básica incompleta, de analfabetos, de población ocupada con ingreso inferior a dos salarios mínimos y de población con carencia por acceso a la seguridad social, lo que se relaciona con la informalidad del mercado de trabajo. Estos aspectos tienen repercusión en los indicadores que ponderan en mayor medida las dimensiones de educación e ingreso, como el IDH o las cifras de pobreza multidimensional, donde Chiapas sigue clasificando en última posición.

Lamentablemente, los indicadores muestran una persistencia de los problemas estructurales detectados en la entidad. Las medidas aplicadas no parecen haber permeado hasta los sistemas de producción y genera- 
ción de riqueza, en buena parte porque los avances se han dado en los aspectos que menos fuerza tienen en la causación circular. Pese a que las advertencias no son nuevas, las soluciones propuestas siguen dirigiéndose a atacar los efectos más que las causas de la marginación, el rezago o la pobreza. El inconveniente de ello es que si la población no es capaz de impulsar por sí misma el proceso de desarrollo, el terreno ganado tenderá a perderse. La dinámica actual, lejos de ser auto-sostenible, requerirá de ayuda exógena continua en forma de flujos crecientes de capital público, no sólo para seguir progresando, sino incluso para consolidar los avances logrados en los últimos años. La alternativa pasa por complementar las actuales políticas con estrategias enfocadas a remover los rezagos históricos que aún prevalecen. Sólo así se podrá desatar una secuencia de verdadero desarrollo integral (e integrador) auto-reforzado.

El impedimento para ello es que la parte del sistema que se está beneficiando de la situación actual carece de incentivos para operar este cambio. Donde hay necesidades, es más fácil establecer relaciones de poder basadas en la dependencia. Así es como la pobreza, la marginación, el rezago y la falta de desarrollo, dentro de ciertos límites, son funcionales al sistema de relaciones desiguales. Las políticas asistenciales evitan que se sobrepasen esos límites, garantizando el mantenimiento del statu quo. La historia reciente de Chiapas es un ejemplo de ello.

\section{BIBLIOGRAFÍA}

Acosta, Félix (2010), «De Pronasol a Oportunidades: política social y persistencia de la pobreza en México», Barataria: Revista Castellano-Manchega de Ciencias Sociales, núm. 11.

Anzaldo, Carlos, y Minerva Prado (2006), Índices de marginación, 2005, México, Consejo Nacional de Población.

Aparicio, Ricardo (2004), Índice absoluto de marginación, 1990-2000, México, Consejo Nacional de Población.

Arriaga, Eduardo, y Kingsley Davis (1969), «The pattern of mortality change in Latin America», Demography, vol. 6, núm. 3. 
Ávila, José Luis, Carlos Fuentes y Rodolfo Tuirán (2001), Índices de marginación, 2000, México, Consejo Nacional de Población.

Barajas, Gabriela (2002), «Las políticas de administración de la pobreza en México: ayer y hoy», Foro Internacional, vol. 42, núm. 1.

BARKIN, David (1972), «¿Quiénes son los beneficiarios del desarrollo regional?», en David Barkin (comp.), Los beneficiarios del desarrollo regional, México, Secretaría de Educación Pública.

Bartra, Armando (2007), «Los municipios incómodos», en Xochitl Leyva y Araceli Burguete (coords.), La remunicipalización de Chiapas: lo político y la política en tiempos de contrainsurgencia, México, Miguel Ángel Porrúa.

Bazdresch, Carlos (1989), La economía mexicana: cuatro ensayos, México, Centro de Investigación y Docencia Económica.

Becker, Gary Stanley (1964), Human capital: a theoretical and empirical analysis, with special reference to education, Nueva York, National Bureau of Economic Research.

Brieger, Pedro (2002), «De la década perdida a la década del mito neoliberal», en AA.VV., La globalización económico-financiera: su impacto en América Latina, Buenos Aires, Consejo Latinoamericano de Ciencias Sociales.

Burguete, Araceli, y Xochitl Leyva (2004), Estudios monográficos: nuevos municipios en Chiapas, Tuxtla Gutiérrez, Consejo Estatal para la Cultura y las Artes de Chiapas / Centro de Investigaciones y Estudios Superiores en Antropología Social.

(2007), La remunicipalización de Chiapas: lo político y la política en tiempos de contrainsurgencia, México, Centro de Investigaciones y Estudios Superiores en Antropología Social / Miguel Ángel Porrúa.

Calva, José Luis (1993), Alternativas para el campo mexicano, México, Fontamara / UNAM.

Carton de Grammont, Hubert (2009), «La desagrarización del campo mexicano», Convergencia, Revista de Ciencias Sociales, vol. 16, núm. 50.

CEja, Concepción (2004), «La política social mexicana de cara a la pobreza», Scripta Nova: Revista Electrónica de Geografía y Ciencias Sociales, vol. 8, núm. 176.

Chenery, Hollis, y Moises Syrquin (1978), La estructura del crecimiento económico: un análisis para el periodo 1950-1970, Oxford, Oxford University Press.

Chesnais, Jean-Claude (1992), The demographic transition: stages, patterns, and economic implications: a longitudinal study of sixty-seven countries covering the period 1720-1984, Nueva York, Clarendon Press.

Coneval (2007), Mapas de pobreza por ingresos y rezago social 2005, México, Consejo Nacional de Evaluación de la Política de Desarrollo Social. 
(2008), Mapas de pobreza por ingresos 2000, México, Consejo Nacional de Evaluación de la Política de Desarrollo Social.

(2011a), Pobreza en México y en las entidades federativas 2008-2010, México, Consejo Nacional de Evaluación de la Política de Desarrollo Social. (2011b), Indice de rezago social; resultados 2010; excel para estados y municipios 2000-2005-2010, y programa de cálculo, México, Consejo Nacional de Evaluación de la Política de Desarrollo Social.

(2013), Resultados de pobreza a nivel nacional y por entidades federativas 2010-2012, México, Consejo Nacional de Evaluación de la Política de Desarrollo Social.

Davis, Kingsley (1956), «The amazing decline of mortality in underdeveloped areas», The American Economic Review, vol. 46, núm. 2.

DOF (2013), «Decreto por el que se emite la Declaratoria de Zonas de Atención Prioritaria para el año 2014», Diario Oficial de la Federación, 3 de diciembre, $5^{\text {a }}$ sección.

El Heraldo de Chiapas (2011), «Chiapas, de los primeros en el combate a la pobreza: Coneval», 30 de julio, en http://www.oem.com.mx/elheraldodechiapas/notas/n2166640.htm (03/07/2014).

(2012a), «La pobreza ya no es más destino para Chiapas; 72 mil dejaron la pobreza extrema», 2 de enero, http://www.oem.com.mx/elheraldodechiapas/notas/n2368770.htm (03/07/2014).

(2012b), «Chiapas dejó 1er lugar de rezago social, respondiendo a la deuda social: JSG», 16 de agosto, http://www.oem.com.mx/elheraldodechiapas/notas/n2658796.htm (03/07/2014).

Frank, André Gunder (1967), «El desarrollo del subdesarrollo», Pensamiento Crítico, núm. 7.

Griffith-Jones, Stephany, y Osvaldo Sunkel (1986), Debt and development crises in Latin America: the end of an illusion, Oxford, Clarendon Press.

Gutiérrez, Humberto, y Viviana Gama (2010), «Limitantes de los índices de marginación de Conapo y propuesta para evaluar la marginación municipal en México», Papeles de Población, vol. 16, núm. 66.

Inegi (1993), XI Censo general de población y vivienda 1990, Aguascalientes, Instituto Nacional de Estadística, Geografía e Informática.

(1994), Chiapas. Resultados definitivos. VII Censo agrícola-ganadero, Aguascalientes, Instituto Nacional de Estadística, Geografía e Informática. (2003), XII Censo general de población y vivienda 2000, Aguascalientes, Instituto Nacional de Estadística, Geografía e Informática. 
(2009), Censo agropecuario 2007, VIII censo agricola, ganadero y forestal, Aguascalientes, Instituto Nacional de Estadística y Geografía.

(2011), Censo de población y vivienda 2010, Aguascalientes, Instituto Nacional de Estadística y Geografía.

Levy, Santiago (1991), Poverty alleviation in Mexico, Washington, World Bank Working Paper Series, núm. 679.

López, Jorge, Bruno Sovilla y Héctor Escobar (2009), «Crisis económica y flujos migratorios internacionales en Chiapas», Revista Mexicana de Ciencias Políticas y Sociales, vol. 51, núm. 207.

López, Jorge, y Baltazar Mayo (2012), «Chiapas, endeudamiento en la encrucijada», Economía Informa, núm. 376.

y Óscar Peláez (2013), «Globalización neoliberal, mercado de trabajo y emigración desde Chiapas», en Jorge López y Óscar Peláez (coords.), Buscando el norte: declive económico y emigración en la región Sierra de Chiapas, México, Plaza y Valdés.

Malthus, Thomas Robert (1984) [1798], Primer ensayo sobre la población, Madrid, Alianza editorial.

Mauss, Marcel (2002) [1924], «Essai sur le don. Forme et raison de l'échange dans les sociétés primitives», l'Année Sociologique, seconde série, en http:// anthropomada.com/bibliotheque/Marcel-MAUSS-Essai-sur-le-don.pdf (21/04/2014).

Mincer, Jacob (1958), «Investment in human capital and personal income distribution», Journal of Political Economy, vol. 66, núm. 4.

Myrdal, Gunnar (1979) [1957], Teoría económica y regiones subdesarrolladas, México, Fondo de Cultura Económica.

Núñez, Violeta (2004), Por la tierra en Chiapas... el corazón no se vence: historia de la lucha de una comunidad maya-tojolabal para recuperar su nantik lu'um, su Madre Tierra, México, Plaza y Valdez.

Ordóñez, Gerardo (2002), La política social y el combate a la pobreza en México, México, unam.

Peláez, Óscar (2012), «Análisis de los indicadores de desarrollo humano, marginación, rezago social y pobreza en los municipios de Chiapas a partir de una perspectiva demográfica», Economía, Sociedad y Territorio, vol. 12, núm. 38.

Pnud (2003), Informe sobre desarrollo bumano México 2002, México, Mundi-Prensa México.

(2008), Indice de desarrollo bumano municipal en México 2000-2005, México, Programa de las Naciones Unidas para el Desarrollo. 
(2012), El indice de desarrollo bumano en México: cambios metodológicos e información para las entidades federativas, México, Programa de las Naciones Unidas para el Desarrollo.

Sánchez, Adolfo (2000), Marginación e ingreso en los municipios de México: análisis para la asignación de recursos fiscales, México, unam / Miguel Ángel Porrúa.

Schultz, Theodore (1961), «Investment in human capital», American Economic Review, vol. 51, núm. 1.

Sen, Amartya (1985), Commodities and capabilities, Amsterdam, North-Holland. TARrío, María, y Luciano Concheiro (2006), «Chiapas: los cambios en la tenencia de la tierra», Argumentos, vol. 19, núm. 51.

Tetreault, Darcy Victor (2012), «La política social y los programas para combatir la pobreza en México, ¿Oportunidades para quiénes?», Estudios Críticos del Desarrollo, vol. 2, núm. 2.

Valenzuela, José (2013), «Un mundo más ancho y más ajeno: neoliberalismo y desigualdades regionales», en Jorge Isaac, Jorge López y Luis Quintana (coords.), Desigualdad y desarrollo regional: Chiapas y el Sur Pacifico mexicano, México, Plaza y Valdés.

VegA, Sergio, Raúl Romo y Ana González (2011), Índice de marginación por entidad federativa y municipio 2010, México, Consejo Nacional de Población.

Zepeda, Eduardo (1994), «El gasto social en Méxicoः de la estabilización ortodoxa al neoliberalismo social», Frontera Norte, número especial: pobreza. 
